\title{
Electro-optic probing of microwave fields using a wavelength-tunable modulation depth
}

\author{
Dong-Joon Lee ${ }^{1}$, Matthew H Crites ${ }^{2}$ and John F Whitaker ${ }^{1}$ \\ ${ }^{1}$ Center for Ultrafast Optical Science and Department of Electrical Engineering and Computer Science, \\ University of Michigan, Ann Arbor, MI 48109, USA \\ ${ }^{2}$ Opteos, Inc. 775 Technology Drive Suite 200, Ann Arbor, MI 48108, USA \\ E-mail: dongjl@umich.edu
}

Received 11 April 2008, in final form 29 July 2008

Published 17 September 2008

Online at stacks.iop.org/MST/19/115301

\begin{abstract}
A design for an electro-optic microwave-field-mapping system that eliminates the need for the polarizer, analyzer and quarter-wave retarder in its optical-probe-beam path, while also exhibiting less laser-induced noise and an enhanced signal-to-noise ratio as compared to conventional single- or double-pass electro-optic intensity modulators, is conceived and demonstrated. The measurement approach utilizes a high-reflection, resonant-microcavity probe, an entirely fiber-enclosed optical-beam path and a photonic heterodyne-mixing technique. A model for the reflective resonant probe is proposed and then employed to calculate electro-optic phase retardation and a modulation-efficiency slope, the latter of which is found to indicate that system sensitivity can be optimized via tuning of the input laser wavelength. This concept is experimentally verified using thin lithium tantalate electro-optic probe crystals configured as balanced resonators, and then an entirely fiber-coupled measurement is demonstrated in a variety of near-field, spatial scans over an X-band patch antenna.
\end{abstract}

Keywords: electric-field measurement, electro-optic effects, electro-optic modulation, Fabry-Perot resonators

(Some figures in this article are in colour only in the electronic version)

\section{Introduction}

Near-field electrical measurement and diagnostic techniques can serve as powerful tools in the design and analysis of highfrequency antennas or circuits where complete field pattern and polarization characterizations would be beneficial. Nearfield information from guided wave or radiating devices has the potential to provide the actual detailed electromagnetic field distributions that conventional scattering-parameter measurements using a network analyzer and on-wafer probing [1] cannot. Although existing microwave near-field scanners have been used for such measurements, their results are typically limited because they are not able to operate nonintrusively in the reactive near-field region of a device-undertest. Furthermore, microwave scanning systems generally must be accompanied by associated numerical post-processing methods to enable a conversion of the measured quantity into an electromagnetic field [2]. This requires one to compute the relative spatial localization of the electric charges and current surface densities directly from the mapped pattern of the probe signals. Ideally, measurements could be made at circuit internal nodes or in the reactive near field at the interior of an antenna, and not via either port-based connections or metallic probes that must be positioned a number of wavelengths from an antenna.

To address these issues, significantly less-invasive, directsensing methods that use photonic-assisted near-field probes and nonlinear-optical techniques have been proposed and implemented. As the most popular of these methods, electro-optic probing (EOP) exhibits a large measurement 
frequency range, low intrusiveness and high spatial resolution [3]. Using probes fabricated purely from dielectric materials and interrogated by optical beams allows for non-contact, non-destructive and high-impedance-yet high-frequencyprobing to be performed at internal nodes of microwave integrated circuits and even within the reactive near field of antennas and arrays.

In order to realize such a variety of beneficial aspects in a measurement method, however, one typically must tolerate a deficiency in some aspect of the relevant technology, and in this case, that would be low field sensitivity. Electrooptic (EO) measurements rely on an extra phase retardation being induced on an optical beam by a low-frequency electric field due to the Pockels effect, typically during a single or double pass of a light beam through the EO medium. The EO sensitivity to electric fields is commonly not much better than $1 \mathrm{~V} \mathrm{~m}^{-1} \mathrm{~Hz}^{-1 / 2}$, which, while often sufficient for nearfield measurements, does not always allow much margin for resolving small signals such as those found in the far field [4].

During typical EO-probing measurements, the optical polarization is oriented at $45^{\circ}$ to the relevant crystal axes of the EO medium, and the EO retardation of the components of the optical beam is converted into an intensity modulation through the function $\sin ^{2}(\Delta \delta)$ (or $\sin (\Delta \delta) / 2$ when using a quarter waveplate in the beam path). This response is created when placing the EO medium between a crossed polarizer/analyzer pair. This modulation function yields an essentially linear slope at $50 \%$ of its transmission maximum, a point that can be attained through the use of waveplates [5]. Since the EO retardation is most often tiny, one can consider that this EOmodulation function has a maximum efficiency slope of 0.5 . In contrast to this so-called sine-squared modulation curve, it has also been demonstrated that an EO-modulation slope can be achieved exclusively through utilization of the FabryPerot effect in the EO medium, i.e., without the need for a polarizer/analyzer pair or a quarter waveplate [6]. In this case, a thin $\mathrm{LiTaO}_{3}$ wafer was employed as a free space, transmissive EO sensor.

The poor maneuverability and opto-mechanical robustness of the free-space EO sensor have been improved upon in several cases through the use of a reflective, fiberbased probe geometry [7-10]. Furthermore, the compactness and reliability of such a fiber-based embodiment provide for enhanced spatial resolution and relief from the necessity of tedious optical alignment.

In this paper, first the modeling of the resonance-based, EO-sensing concept is extended so that it applies to reflective EO probing. In this case, the addition of extra distributed Bragg coatings on the faces of the EO medium is considered, and the operating location on the modulation-efficiency slope is found to be controllable over a significant range through tuning of the laser wavelength. Besides the sensitivity enhancement, the resonance-based reflective geometry has an advantage in reducing laser-induced noise, as it needs to deliver fewer photons to a photodetector in order to achieve the equivalent amount of EO-modulation signal.

Second, experiments have been conducted to highlight the enhanced signal-to-noise characteristics that are possible using

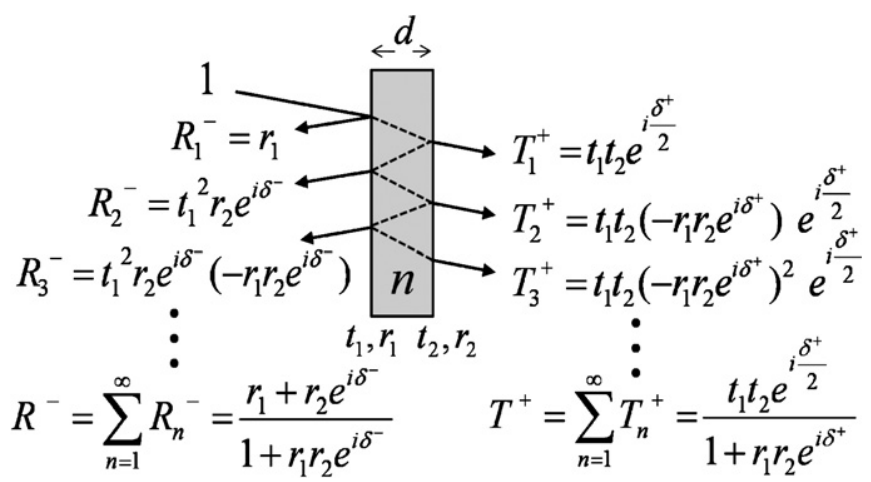

Figure 1. Interferometric field components of transmission and reflection from the Fabry-Perot effect in an EO wafer. $\left(r_{1,2}\right.$ and $t_{1,2}$ : Fresnel reflection/transmission field coefficients at the front, or incident, and back interfaces. The incident beam is drawn with a non-normal incidence angle for ease in separating the transmitted and reflected beams.)

thin EO crystals, and the ability to produce a less-complicated EOP system by eliminating the polarizer/analyzer pair and the optical-biasing, quarter waveplate of traditional intensitymodulating probes is demonstrated. Finally, the utility of this novel system is shown to reach high-frequency field sensing through the application of a photonic down-conversion technique.

\section{Theoretical modeling}

The modeling of a general, resonance-based EOP system was first described in our previous work on a transmissive system [6]. Here, the same methodology is adopted for the quantitative analysis of reflective systems. In EOP systems, a single plate or layer of EO material can act as a FabryPerot etalon in which refractive-index discontinuities lead to multiple internal reflections and, subsequently, multiple cavity-transmission components. The index of refraction, $n$, and the thickness of the EO medium, $d$, determine a roundtrip optical phase delay of the etalon, $\delta=4 \pi n d / \lambda$. When one includes the Pockels effect for the EO sensor, the index is modified to $n \pm \Delta n$ in the presence of a static electric field, causing an additional single-pass phase delay, $\Delta \delta=$ $2 \pi \Delta n d / \lambda$. As a result, the single-pass phase delay (i.e., in transmission), including an electric-field-induced EO effect, can be written as $\delta^{ \pm} / 2=\delta / 2 \pm \Delta \delta$, where the \pm indicates the sign of the phase retardation depending on the light direction and polarity of the applied electric field. Thus, as a lowfrequency electric field is applied along the $c$-axis of an EO wafer, a transmitted optical beam experiences an additional single-path phase retardation, $\Delta \delta$, and the field transmission and reflection will be modified to $T^{+}$and $R^{-}$, as illustrated in figure 1. It should also be noted that the $T^{+}$and $R^{-}$have the opposite signs of EO shift due to their opposite propagation directions.

As the refractive index of an EO etalon is changed by an electric field, $E$, the original power transmittance, $I_{\mathrm{t}}(\lambda)=$ $\left|T(\lambda)^{2}\right|$, and reflectance, $I_{\mathrm{r}}(\lambda)=\left|R(\lambda)^{2}\right|$, at wavelength $\lambda$ are shifted to $I_{\mathrm{t}}(\lambda, E)=T^{+}(\lambda, E)^{*} T^{-}(\lambda, E)$ and $I_{\mathrm{r}}(\lambda, E)=$ 


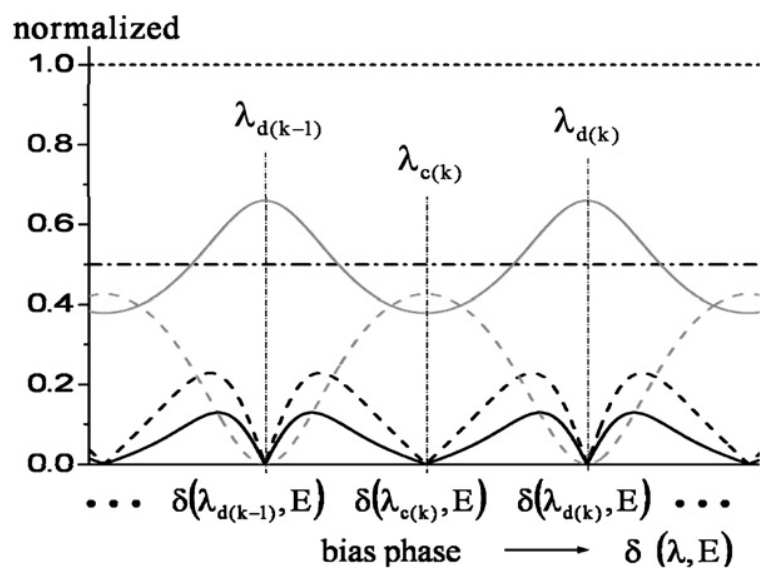

Figure 2. Simulations for a reflective, resonance-based, $\mathrm{LiTaO}_{3}$ EOP system (over two etalon modes; no coatings). All quantities plotted versus phase, which varies as a function of laser wavelength and the applied low-frequency electric field. Dashed gray line: normalized reflectance $I_{\mathrm{rn}}$; dashed line: spectral slope $\left|\mathrm{d} I_{\mathrm{rn}} / \mathrm{d} \delta\right|$; solid black line: normalized EO signal $I_{\text {EOn }}$; dotted line: normalized EO-phase retardation for ideal double-pass case $2 \Delta \delta$; solid gray line: relative EO-phase retardation versus ideal double pass case $\angle I_{\mathrm{r}} /(2 \Delta \delta)$; dash/dot line: $I_{\text {Eon }}$ for ideal double-pass case.

$R^{-}(\lambda, E)^{*} R^{+}(\lambda, E)$ due to the EO-phase retardation. The amount of the transmittance (or reflectance) change, $\Delta I_{\mathrm{t}(\mathrm{or}, \mathrm{r})}(\lambda, E)$, strongly depends on the minute phase change $\Delta \delta(\lambda, E)$ that accompanies the bias wavelength and the applied field. The optical-beam wavelength determines the static operating bias phase, whereas the electric field causes an extra, EO-induced phase. In other words, the wavelength sets the amount of static transmission (or reflection) depending on the spectral response of the etalon, and the amount of transmitted (or reflected) modulation intensity is determined by the slope of the spectral response at the operating wavelength, along with the amount of the EO-phase modulation. The spectralresponse slope, $\mathrm{d} I_{\mathrm{t}(\text { or, r })}(\lambda, E) / \mathrm{d} \delta(\lambda, E)$, presents the EOtransmission (or reflection) sensitivity versus minute changes in the phase and yields the wavelengths that maximize the resonance-based EO signal.

The EO-phase retardation for transmission and reflection can be expressed as $\angle I_{\mathrm{t}}=\angle T^{+}-\angle T^{-}, \angle I_{\mathrm{r}}=\angle R^{-}-\angle R^{+}$, respectively [11-13]. These are identical to each other and both have an in-phase (or out-of-phase) shape of the spectral response as in light transmittance (or reflectance). The product of the spectral slope and the amount of EO-phase retardation governs the total EO-signal strength. Simulations of the reflectance and the spectral slope versus minute phase perturbations for a $\mathrm{LiTaO}_{3}$ probe $(n=2.18)$ are presented in figure 2, along with the relative EO-phase retardation and signal strength. These quantities are also compared to an ideal, conventional, non-resonant, double-pass EOP configuration with the same crystal, but with a $100 \%$ high-reflection (HR) coating placed on the second interface (interface 2 in figure 1).

Analyzing figure 2, the normalized EO-signal strength in the reflective, resonance-based system is $I_{\mathrm{EOn}}=\left|\mathrm{d} I_{\mathrm{rn}} / \mathrm{d} \delta\right| \times$ $\angle I_{\mathrm{r}} /(2 \Delta \delta)$, and it is observed to have up to $\sim 26 \%$ of the EO signal in the ideal, conventional double-pass system. Since $I_{\text {EOn }}$ is a normalized relative quantity for input-beam intensity

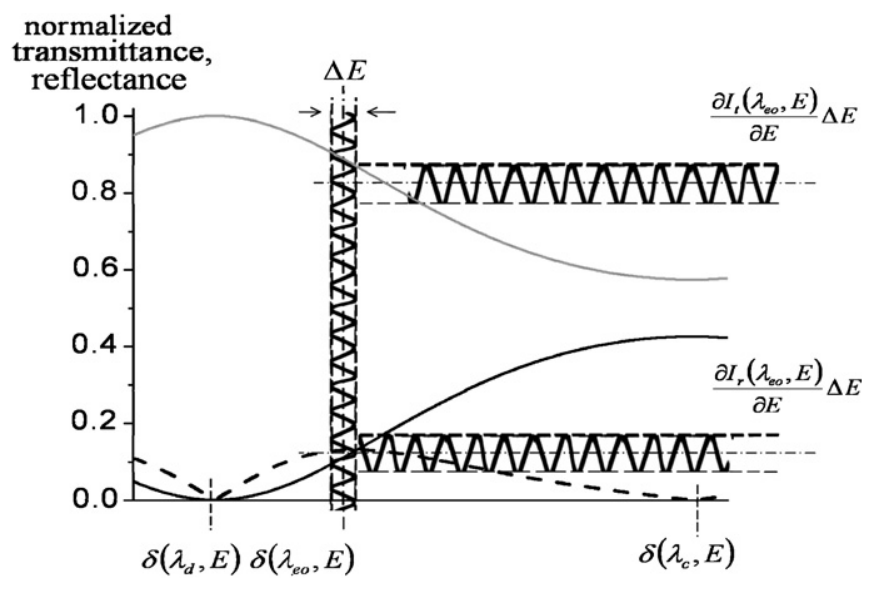

Figure 3. Principle of resonance-based EO-amplitude modulation for transmission and reflection cases (a half mode for a $\mathrm{LiTaO}_{3}$ etalon with no reflective coatings). Gray line: normalized transmittance; solid black line: normalized reflectance; dashed black line: EO-signal strength. Both modulations (exaggerated for ease of observation) have the same amplitude with opposite phase.

$\left(I_{\text {in }}\right)$ and double-pass phase retardation $(2 \Delta \delta)$, the absolute intensity modulation is expressed as $I_{\mathrm{EO}}=I_{\mathrm{EOn}} \times\left(2 \Delta \delta I_{\text {in }}\right)$. As the slopes and EO phase retardation for transmission and reflection are respectively out-of-phase $\left(\mathrm{d} I_{\mathrm{rn}} / \mathrm{d} \delta=-\mathrm{d} I_{\mathrm{tn}} / \mathrm{d} \delta\right)$ and identical $\left(\angle I_{\mathrm{t}}=\angle I_{\mathrm{r}}\right)$, the EO strengths (i.e., the product of slope and retardation) will be the same for the two cases of the opposite phase and for any wavelength, even though the transmitted and reflected light are complementary $\left(I_{\mathrm{rn}}=\right.$ $\left.1-I_{\mathrm{tn}}\right)$. This indicates that the reflection configuration for EOP systems has the advantage of requiring a lower intensity of light in order to achieve the same EO modulation as the transmissive embodiment. Consequently, there will also be less laser-induced noise in the modulation and the electricfield measurement (figure 3).

\section{Experimental demonstration}

An experimental setup that demonstrates the fiber-based amplitude and phase sensing of an RF electric field without the need for conventional, intensity-modulation polarization components is shown in figure 4 . The fiber-based polarization controller unit sets the incident beam polarization to maximize the induced EO-phase retardation associated with the EOprobe crystal. A commercial, distributed-feedback laser diode operating at $\sim 1305 \mathrm{~nm}$ at room temperature supplied $3 \mathrm{~mW}$ of optical power for interrogating the tangential electric field above a microstrip-transmission-line serving as the device under test (DUT). The $0.02 \mathrm{~nm}$ linewidth and $\sim 0.1 \mathrm{~nm}{ }^{\circ} \mathrm{C}^{-1}$ of temperature tunability of the laser-enabled spectral responses of various thin crystals to be observed versus wavelength with acceptable resolution.

The structure of the fiber-based resonant EO probe is also shown in figure 4 . The approximately index-matched interfaces between the fiber, ferrule, AR coating and UVcuring adhesive (with $n \sim 1.5$ ) minimized the discontinuities within the beam path and provided a low-optical insertion loss to the probe/etalon. While the resonant-reflection probe 


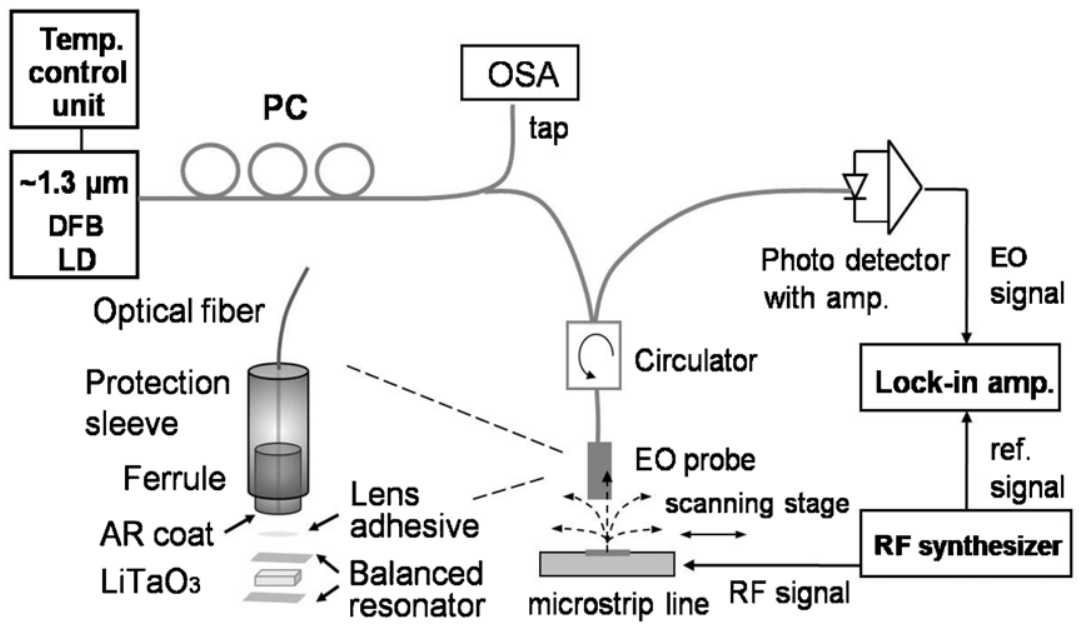

Figure 4. Experimental setup of the all-fiber, resonance-based, EO-probing system (DFB LD: distributed feedback laser diode, PC: polarization controller, OSA: optical spectrum analyzer). The gray and black lines are optical fibers and electrical connections, respectively. The dashed lines on the DUT are electric-field flux.

had its maximum EO-modulation response at a low reflected intensity (bottom curves in figure 3), a conventional, doublepass configuration reflects a high intensity from an HR coating on the exposed face of a probe. In the latter, a GRIN lens would be used for collimating or focusing the probe beam into a relatively thick crystal $[8,9]$, while in the former, a thin crystal was mounted directly on the fiber facet (as in $[7,9,10]$ ). In the non-resonant configurations, the quarter waveplate/analyzer polarization components would be required, and typically $50 \%$ of the input probe light delivered to the detector, about five times more than for the resonant probe. While a non-resonant system can be operated with power levels reduced from this $50 \%$ level [14-16], a greater benefit in signal-to-noise ratio can be realized with the steeper modulation slopes of the resonant techniques. Moreover, in non-resonant, conventional systems, the index mismatch between the fiber/ferrule glasses and the crystal causes detrimental spectral modulation. As a consequence, the trade-off for achieving greater reflection is an increase in the laser-induced noise, or one could say that laser noise is, in practice, more efficiently suppressed in the reflective, resonance-based cases.

In our test experiment, a 50-55 $\mu \mathrm{m}$ thick, x-cut $\mathrm{LiTaO}_{3}$ wafer was used for the EO probe, since this etalon allowed a full-resonant mode in reflected or transmitted intensity to be observed when tuning over several $\mathrm{nm}$ of wavelength. This resonant EO probe was configured to sense the tangential EO amplitude and phase signal for the DUT, which was fed with a $23 \mathrm{dBm}$ sinusoidal input at $3 \mathrm{MHz}$. The microstrip line was scanned laterally under the fiber-based EO probe, revealing the field pattern above and to the sides of the top metal strip. At the middle of the microstrip, where the phase was observed to change by $180^{\circ}$, the amplitude dropped to the noise floor, and a signal-to-noise ratio of $\sim 80 \mathrm{~dB}$ was found with the wavelength at the maximum spectral bias in the half-mode window.

To characterize the strong spectral dependence of the EO sensitivity, the crystal was positioned so that it intersected the strongest tangential electric field, near the edge of the microstrip top electrode. The EO signal was then recorded every $0.1 \mathrm{~nm}$ as the wavelength was tuned, utilizing an uncoated crystal as the etalon. Figure 5(a) shows results of the calculated and measured reflectance, as well as the measured EO signal versus wavelength. These signal levels are stated in the RF power levels that are present on a lock-in amplifier at the output of the photodetector, which demodulates the EO-signal amplitude and phase from the probe light beam. As expected, the signal completely disappeared twice, at the destructive $\left(\lambda_{\mathrm{d}}\right)$ and constructive $\left(\lambda_{c}\right)$ wavelengths, and the EO response was found to be maximum at a relatively low reflected intensity. Experimental results show slightly less spectral resonance than the simulation, since the first round-trip component from the etalon that contains EO retardation couples back to the fiber core with slightly less efficiency than expected.

To make the resonance-based EOP system more sensitive, the spectral slope was increased through the judicious use of reflective coatings on the crystal surfaces. To build a balanced resonator, we evaporated three alternating dielectric layers of $\mathrm{ZnSe}$ and $\mathrm{MgF}_{2}$ at quarter-wavelength thickness $\left(\mathrm{ZnSe} / \mathrm{MgF}_{2} / \mathrm{ZnSe}\right)$ on both surfaces of a second $\mathrm{LiTaO}_{3}$ plate. Again, because higher-order round-trip components get coupled back to the fiber less efficiently, the HR coatings were found to each yield $r \sim 0.63$ of the Fresnel field reflection coefficient (figure 5(b)), even though $r \sim 0.82$ was expected. Adding another pair of $\mathrm{MgF}_{2} / \mathrm{ZnSe}$ coatings on each face of a third EO plate yielded an experimental $r$ of $\sim 0.72$ (figure 5(c)).

The balanced probes with three and five layers of coatings enhanced the EO signal level approximately five and ten times compared to that of the bare probe, respectively, as seen in figures $5(b)$ and $(c)$. However, the bandwidths of the etalon modes were reduced to $63 \%, 33 \%$ and $23.3 \%$ for the three probes with respect to the full (i.e., 100\%), flat bandwidth in a non-resonant case. Since the EO wafers used have a $10 \%$ thickness tolerance as specified by the manufacturer, the mode separation and resonance wavelengths $\left(\lambda_{\mathrm{d}}, \lambda_{\mathrm{c}}\right)$ are positioned at different wavelengths. Assuming the same thickness for each probe sample before coating, the 

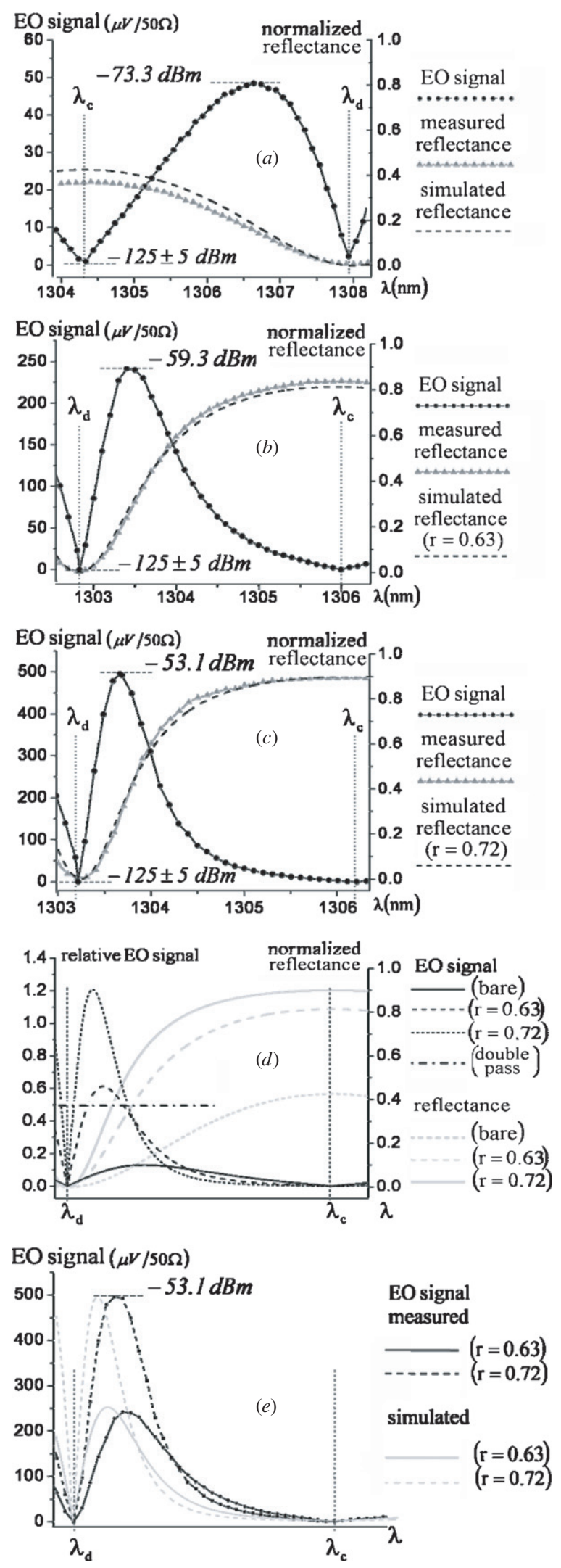

Figure 5. EO-signal strength and reflectance from probes with various resonant conditions: $(a)$ bare wafer; $(b)$ three layers of balanced HR coatings; $(c)$ five layers of balanced HR coatings; (d) comparison of computed results for the three probes;

(e) normalized comparison of measured and simulated EO signals in (b)-(d) calculated relative EO-signal strengths and reflectances for the Fresnel field reflections of the three etalons are displayed in figure $5(d)$. The maximum normalized EO signal level that can be achieved from the conventional double-pass, non-resonant configuration is 0.5 , and the resonant probes with three and five layers of dielectric HR coatings show enhancement factors of $\sim 1.2$ and 2.4 times this level, respectively. A comparison of the normalized measured and simulated EO signals in the etalon probes are shown in figure $5(e)$. The values are compared at the referenced common $\lambda_{d}, \lambda_{c}$ for convenience. The measured peak EO signals are observed farther from $\lambda_{d}$ than in the simulation cases. This is due to the imperfections in the resonator, particularly at $\lambda_{\mathrm{d}}$ where, in practice, some amount of light transmission greater than zero exists. This minimum-transmission offset not only indicates the quality of the resonator, but also impacts the shape of the EO-signal plot. Further numerical and quantitative analysis is to be discussed in a separate publication.

Stronger resonant cavities not only enhance the signal strength, but also improve noise quality by requiring less light be delivered to the detector, as discussed previously. For figure $5(c)$, the peak EO signal of $-53.1 \mathrm{dBm}$ at $3 \mathrm{MHz}$ was observed on the lock-in when only $\sim 20 \%$ of the $3 \mathrm{~mW}$ input power was used. A simple increase in input power to $7 \mathrm{~mW}$ results in $7.3 \mathrm{~dB}$ of additional EO-signal enhancement, although further improvement was limited by the laser power and photodetector saturation. As the wavelength approaches $\lambda_{d}$, the signal per unit reflected power will increase significantly. Hence, further signal enhancement would be feasible with high power versions of the laser and detector. An alternate option for signal enhancement is improving the resonator $Q$ factor through thermal expansion of the fiber core, which improves the coupling efficiency $[7,9]$.

\section{Application to high-frequency sensing}

The $\geqslant 80 \mathrm{~dB}$ signal-to-noise ratio enables measurement of high-frequency signals with wide dynamic range, although generally the noise level tends to increase with input frequency due to electromagnetic interference between the DUT, the photodetector and the read-out instruments used. Still, lownoise, high-speed detectors make direct high-speed sensing with cw lasers feasible [17]. This is because the resonancebased probes that are made of thin crystals maintain an essentially instantaneous cavity lifetime for the photons in the resonator, even for reasonably high-frequency-signal sensing $(>10 \mathrm{GHz})$. In structures with higher $Q$ that might be considered for even greater enhancement of the EO-phase retardation, the photons reside for a longer time, reducing the bandwidth $[11-13,18]$.

In addition to a sensitivity enhancement, the all-fiber configuration of figure 4 provides an improved spatial resolution and scanning mobility for field mapping compared to free-space measurement systems. This is due to the small optical mode-field diameter and the flexibility realized by mounting the electro-optic field sensor at the end of an optical fiber [7-10]. 


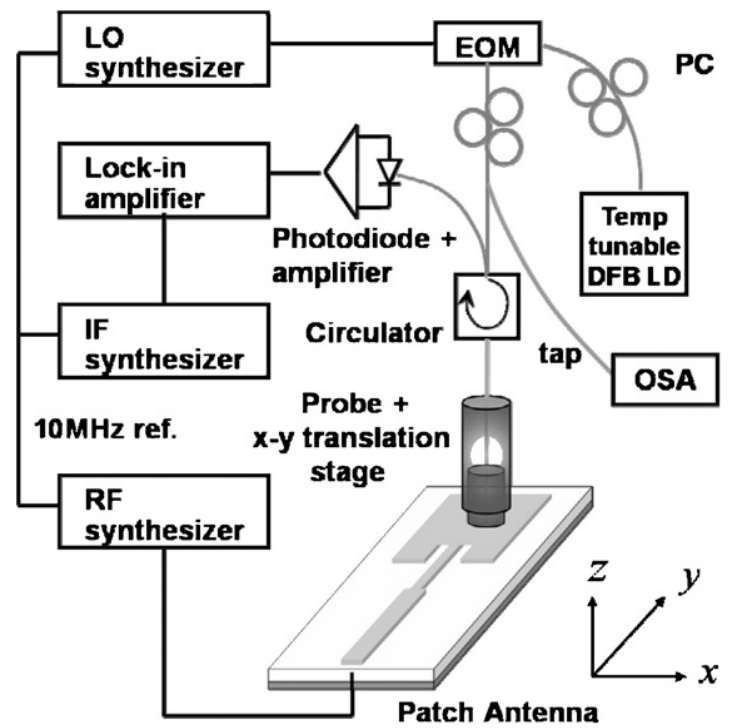

Figure 6. Experimental setup of the all-fiber, resonance-based, photonic-heterodyne-down-conversion, EO-probing system using modulated cw light. (The gray and black lines are optical fibers and electrical connections, respectively.)

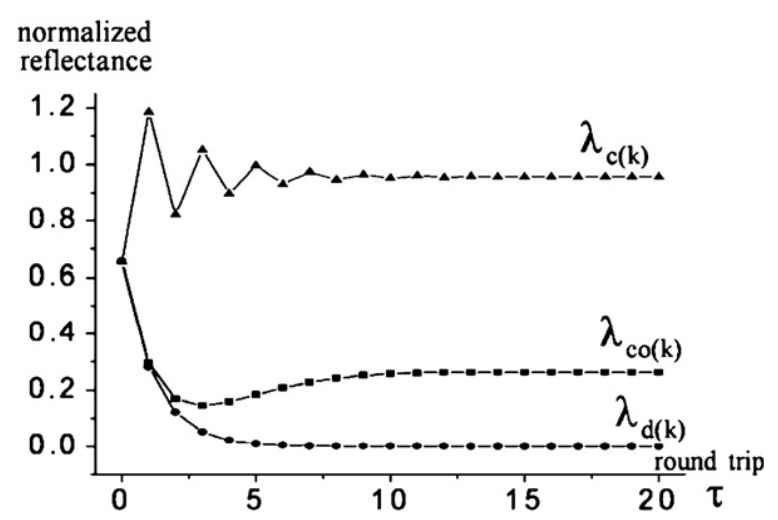

Figure 7. Transient reflectance responses of a balanced resonator $(r=0.81)$ versus photon round-trips in the etalon at constructive, peak-EO-signal and destructive wavelengths, respectively $\left(\lambda_{\mathrm{c}}, \lambda_{\text {eo }}\right.$ and $\lambda_{\mathrm{d}}$ ).

Despite these advantages, most high-frequency EOP systems have required either expensive pulsed lasers $[3,8,9,12,13,19]$ or $\mathrm{cw}$ lasers coupled with fast photodetectors $[7,17]$ for their operation. To realize a fast EOP system with a slow detector, we have extended the resonance-based methodology, with its simplified beam path, to high-frequency sensing using a photonic-mixing technique that down-converts high-frequency information into the lowfrequency band of a slow detector. Unlike the harmonicmixing technique $[3,8,19]$, which utilizes the harmonics of the repetition rate of a pulsed laser as a local oscillator (LO), a method proposed by Kuo [20] and improved by Sasagawa [21] has been adopted. Here, a cw laser is modulated by a conventional electro-optic modulator (EOM), which imparts a sideband onto the optical beam for use as an LO to mix down, within the EO probe, the signal frequency to an intermediate frequency (IF). Combining this mixing process with the cavityenhancement effect, we present, to our knowledge, the first

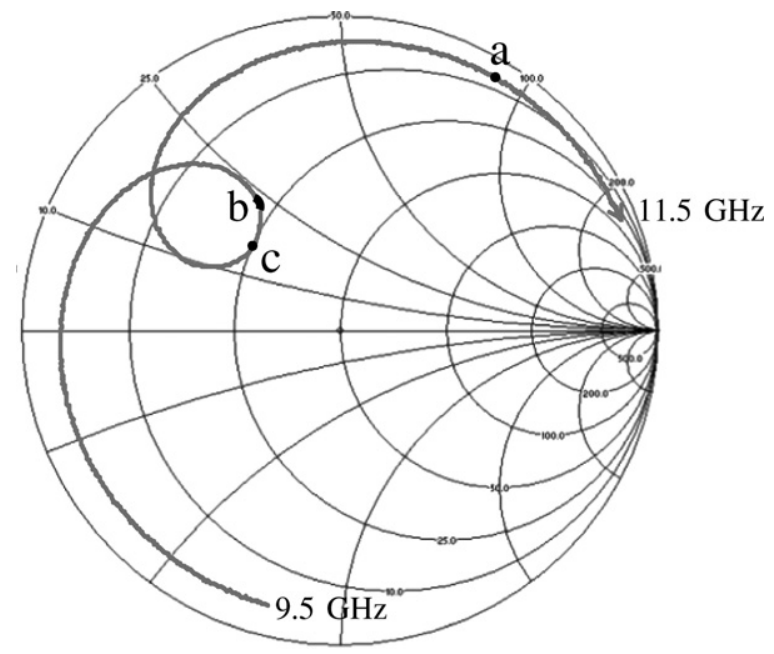

Figure 8. $S_{11}$ of the DUT patch antenna. (Point a: highly reflective point at $11.26 \mathrm{GHz}$; point $\mathbf{b}$ : narrow band close to the minimum return loss at $10.438-10.446 \mathrm{GHz}$; point $\mathbf{c}$ : minimum return loss at 10.485 GHz.)

use of an all-fiber enclosed, resonance-based, heterodynedownconversion, EO-sensing embodiment.

Figure 6 displays the experimental setup of this new EO field-sensing implementation. An inexpensive, fiberpigtailed, temperature-tunable, $\mathrm{cw}$ laser diode (now at the more common telecommunications wavelength of $1560 \mathrm{~nm}$ ) is coupled with the resonant EO sensor, resulting in a significant simplification of the conventional EO optical-polarizationcontrol configuration. That is, the polarizer/analyzer pair and the quarter waveplate employed for nearly all other EOmodulation sensors are eliminated. Modulated laser light (with average power of $8 \mathrm{~mW}$ ) was delivered to a $\mathrm{LiTaO}_{3}$ probe (balanced 5 layer coating optimized for $1560 \mathrm{~nm}$ ) having $r=$ 0.81 , and the EO-modulated beam returning from the probe was routed to the detector through a fiber-optic circulator. The experimental EO signal peak is observed at a wavelength 'bias' of $1562.1 \mathrm{~nm}$, where it experiences the highest slope of the modulation response. As the wavelength was shifted away from this maximum slope and the destructive wavelength, $\lambda_{\mathrm{d}}$, the EO signal was observed to decrease due to the reflection slope becoming more shallow, while noise increased in response to the increased reflected light intensity. The signal and noise levels converge to a finite value as the slope flattens.

Figure 7 suggests the limits of the high-speed response of the resonant $\mathrm{EO}$ probe, as determined by the increasing photon lifetime in the EO etalon with cavity quality factor. The figure is generally valid for an arbitrary thickness of the resonator with balanced reflections of $r=0.81$. The temporal response shows heavy spectral dependence based on the resonance conditions. For instance, with crystal thickness $d$, the roundtrip phase delay is $\delta=4 \pi n d / \lambda$, a function of wavelength which, by its presence in the exponent of the term, $\exp (\mathrm{i} \delta)$, governs the duration and shape of a transient response as well as a steady-state response. The transient responses typically converge to the steady-state values after several round-trip 


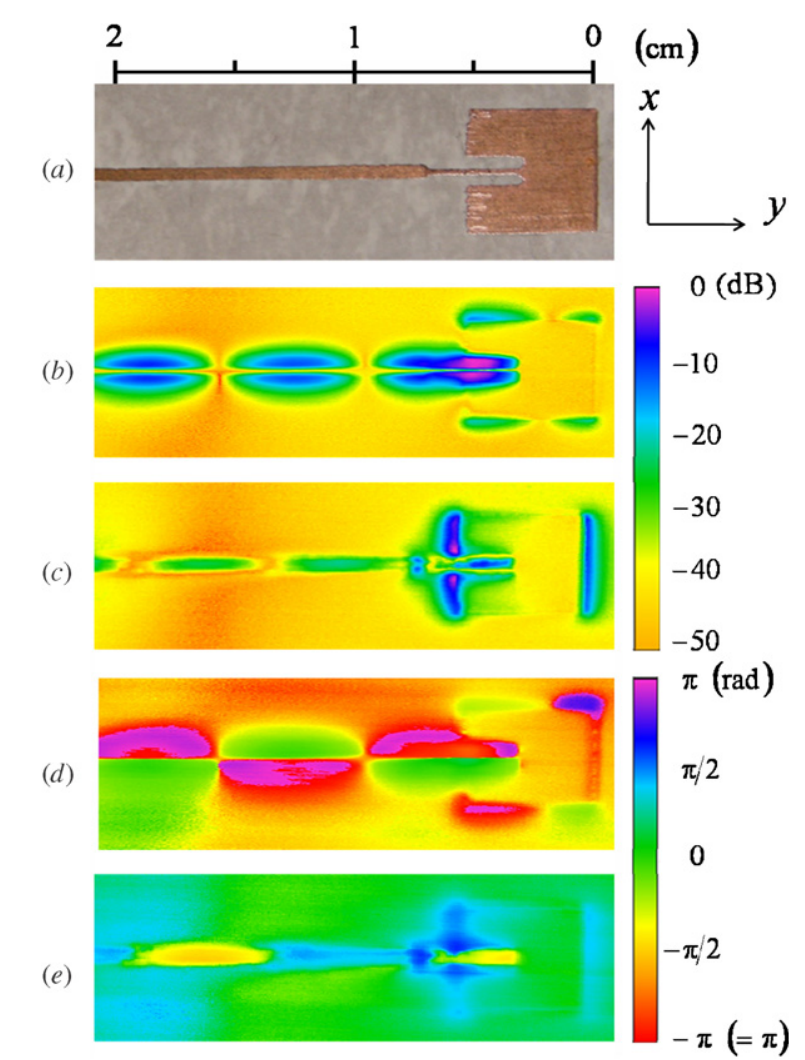

Figure 9. Tangential near-field patterns of a patch antenna at point a (log scale). (a) Photograph and dimension of the DUT;

(b) amplitude of $x$ and (c) $y$ electric-field components; $(d)$ phase of $x$ and $(e) y$ components. delays, $\tau$, although the duration of the transient response has heavy spectral dependence. For the $52 \mu \mathrm{m}$, resonant $\mathrm{LiTaO}_{3}$ EO probe, the $\tau$ value is $756 \mathrm{fs}$, and the first five to seven round trips are sufficient to nearly reach the full interferometric condition at the peak EO signal wavelength, $\lambda_{\text {eo. }}$. This timescale $(\sim 4-5 \mathrm{ps})$ is sufficiently instantaneous to sample $100 \mathrm{ps}$ of a high-speed periodic signal, such as a $10 \mathrm{GHz}$ RF sinusoid, with high fidelity. It is within this microwave X-band range that we demonstrate the system and the resonant probe of figure 6 .

Numerous field-mapping scans were conducted on a recessed-feed patch antenna with a quarter-wavelength transformer to facilitate impedance matching, and the patterns captured show how the electric near-field distribution can be used to examine the quality of the impedance match and the aperture-level field behavior. To first identify the resonance characteristics of the antenna, the scattering parameter $S_{11}$ was measured over a $2 \mathrm{GHz}$ bandwidth on a network analyzer (figure 8). The minimum return loss was determined to be $9 \mathrm{~dB}$ at a frequency of $10.485 \mathrm{GHz}$ (point c).

For the EO characterization, we first measure an arbitrary, highly mismatched frequency (point a, at $11.26 \mathrm{GHz}$ ) where a VSWR of 32.5 was expected to yield a strong standing-wave pattern. To measure $11.26 \mathrm{GHz}$, the $\mathrm{cw}$ laser wavelength is set at the most efficient bias wavelength $(1562.1 \mathrm{~nm})$ and modulated via the external EOM at $11.263 \mathrm{GHz}$. This provided a $3 \mathrm{MHz}$ IF signal after the optical sideband was mixed with the microwave signal in the EO probe. The resultant measured tangential components of the near electric field at the DUT

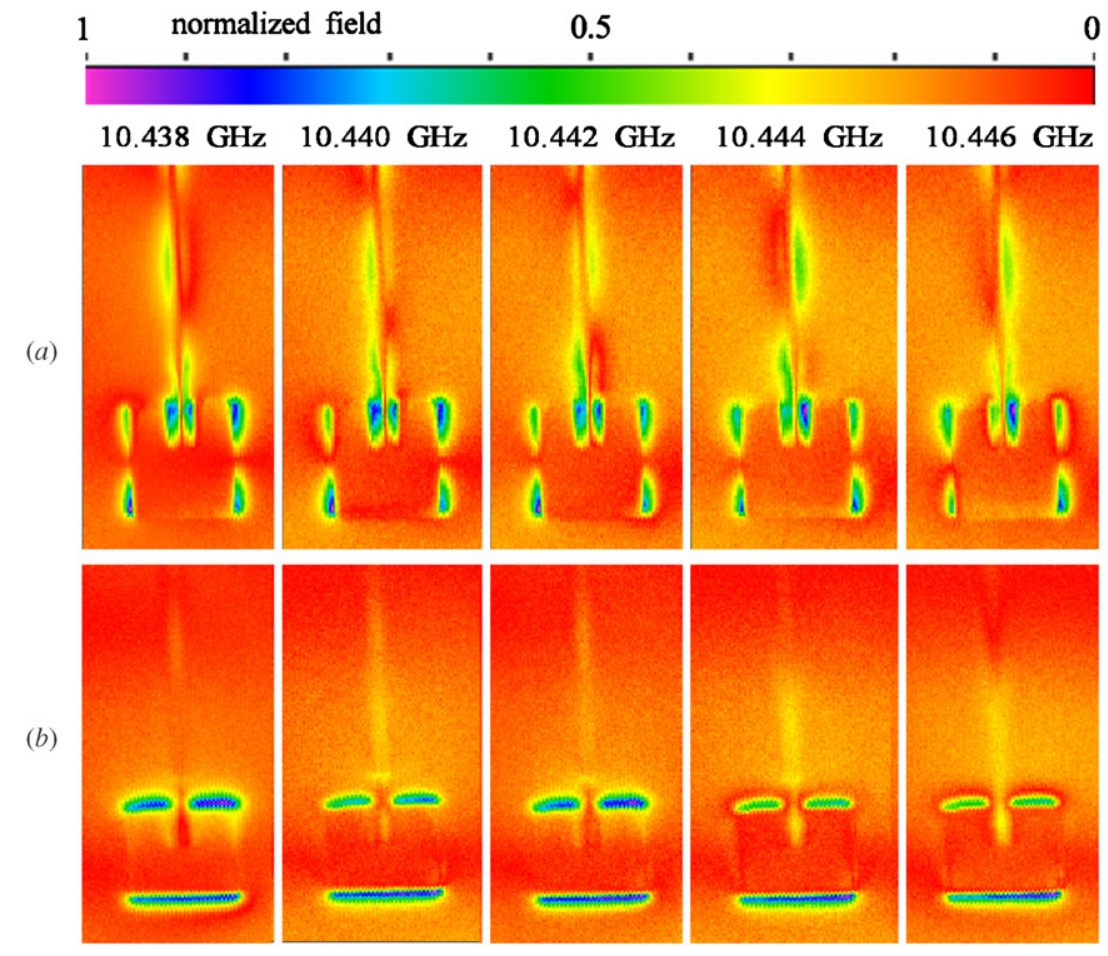

Figure 10. Tangential electric near-field patterns of a patch antenna at band $\mathbf{b}$ (normalized linear scale, $0.1 \mathrm{~mm}$ scanning resolution). (a) Evolution of $x$ component and $(b) y$ component of electric field versus frequency. 


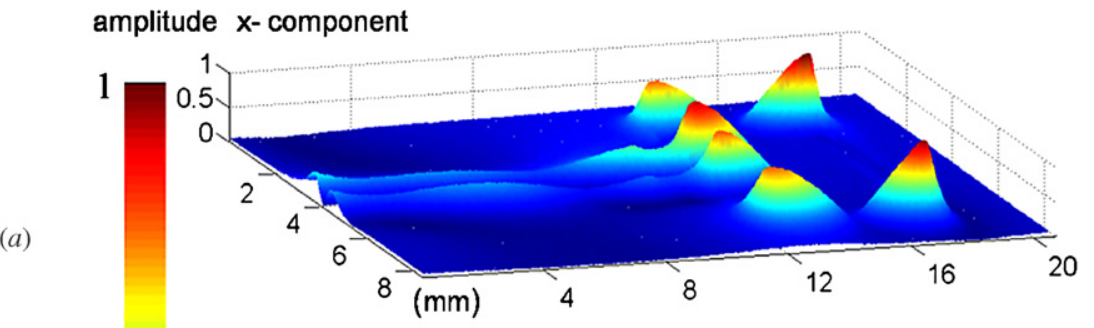

$(a)$

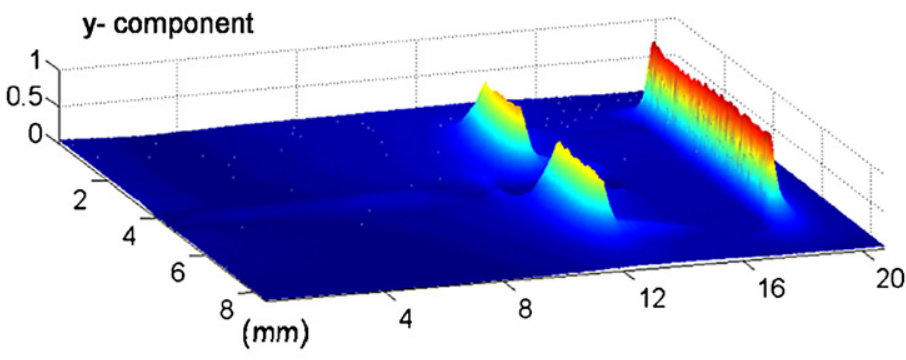

0

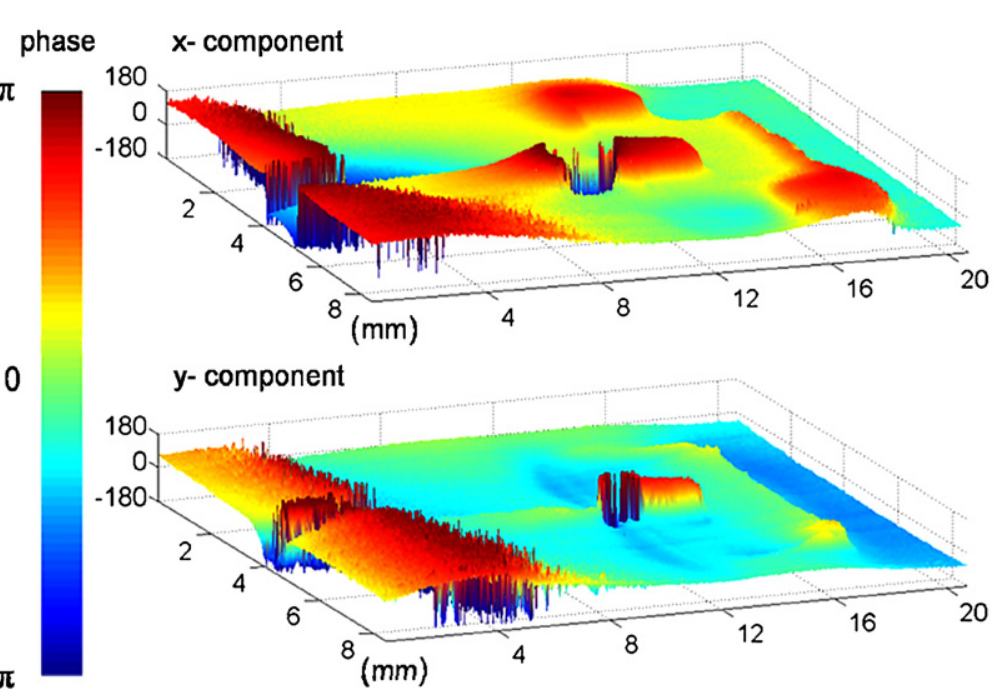

Figure 11. Tangential near-field patterns of a patch antenna at point $\mathbf{c}$ (normalized linear scale, $40 \mu \mathrm{m}$ scanning resolution). (a) Electricfield amplitudes and $(b)$ phases, for both $x$ and $y$ components (noisy part results from loss of phase lock at low-signal amplitude).

frequency of $11.26 \mathrm{GHz}$ are presented in figure 9, along with a photograph of the actual DUT.

The scan area is $22 \mathrm{~mm} \times 8 \mathrm{~mm}$ and a $40 \mu \mathrm{m}$ step resolution was used. With such fine scanning steps and a $10 \mu \mathrm{m}$ spatial resolution for the probe, a sampling area of much less than $100 \mu \mathrm{m}$ was realized, and the probe easily resolved the $200 \mu \mathrm{m}$ width of the narrow transformer line. Figures $9(b)$ and $(c)$ show two orthogonal tangential electric-field amplitude distributions at the highly mismatched frequency of point $\mathbf{a}$. The probe is nearly $50 \mu \mathrm{m}$ above the DUT, which is fed with $+15 \mathrm{dBm}$ of RF power. As expected, the majority of the input power is not delivered through the matching line and is reflected back to create a strong standing wave. This is observed in the $x$ and even $y$ components as a higher-order mode. Each vector field component shows a $50 \mathrm{~dB}$ SNR and the absolute EO-signal-peak point is $-36.1 \mathrm{dBm}$ for the $x$ component. The phase, also shown in figures $9(d)$ and $(e)$ alternates for each standing-wave peak by $180^{\circ}$, and the $5 \mathrm{~mm}$ length of each standing-mode cycle, partially residing in the substrate, is a reasonable spacing for the $11.26 \mathrm{GHz}$ input.

The standing pattern decreases significantly as the input frequency approaches the antenna resonance. For instance, the field distributions for the narrow band (10.438-10.446 GHz, VSWR 2.7) at point $\mathbf{b}$ are shown in figure 10 . The patch DUT exhibits a stronger and more distributed field pattern for the guided signals, while supporting a diminished standing wave. In addition, the relatively rapid transition over the circular-resistance and parabolic-reactive traces on the Smith chart indicates large impedance changes even over the narrow band.

The $S_{11}$ trace on the chart contains strong impedance evolution versus frequency. In other words, the amplitude and phase of the reflection, $S_{11}$, is only another transformation of impedance which controls the frequency-dependent signal flow. Although the frequency-dependent device impedance, $Z(\omega)$, governs the radiation characteristics of the antenna, the $S_{11}$ (or $\left.Z(\omega)\right)$ trace from a network analyzer measurement is 
not enough to explore the detailed, impedance-dependent, full near-field distributions.

The evolution of the field distribution versus the sampled frequencies in band $\mathbf{b}$ is also demonstrated in figure 10. The change in symmetry and the variation in signals peaks on the feed line are noted, along with the fact that such a detailed high-frequency distribution is virtually impossible for a conventional finite-element-based simulation method to compute quickly.

The radiation pattern has the lowest return loss at $10.485 \mathrm{GHz}$ (point c), and the near-field scan is presented in an oblique view in figure 11 . The most efficient radiation condition does not necessarily promise a uniform distribution of the electric field over the DUT. Since the near field is the fundamental basis of the far-field pattern, the near field can be a crucial clue to the mid- and far-field evolution of radiation. Asymmetric distributions in the near field could cause undesired beam steering and far-field distortion. Such non-uniform fields can be adjusted more evenly by fine frequency tuning, as seen in the $10.442 \mathrm{GHz}$ example shown in figure 10.

Furthermore, this near-field characterization is useful for planar arrayed antenna designs. The interplay of phased array elements can modify whole-beam-pattern parameters such as directivity or steering. For instance, imaging the patterns of figure 10 as a five-arrayed-antenna set, the analysis of each unit antenna can be a strong tool for evaluating the desired radiation performance of the whole antenna set.

\section{Conclusion}

We have developed a resonance-based, fiber-optic EO probe that has been demonstrated to provide enhanced sensitivity over conventional double-pass EO probes. An EOP system based on such probes can eliminate the need for the standard analyzer/waveplate sensing configuration and provide improved noise performance by limiting the light intensity needed for photodetection. The signal-to-noise characteristics can be further increased by enhancing the resonant performance of the EO probes. Through utilization with a photonic heterodyne-down-mixing technique, the resonant EO concept was applied to a practical, highfrequency, electro-optic sensing system that is suitable for scrutinizing RF near fields in order to evaluate the performance of microwave devices. Using a low-cost, cw-pigtailed laser diode and an all-fiber-guided embodiment, the resonancebased probe provides reliable and simplified optical-system realization.

\section{Acknowledgment}

This work was supported through a subcontract from Opteos, Inc., under the AFOSR STTR program, contract number FA9550-04-C-0079.

\section{References}

[1] Godshalk E M 2000 Characterization of surface mount packages at microwave frequencies using wafer probes IEEE MTT-S Int. Microwave Symp. Digest pp 1887-90

[2] Yaghjian A G 1986 An overview of near-field antenna measurements IEEE Trans. Antennas Propag. AP-34 30-45

[3] Yang K, Marshall T, Forman M, Hubert J, Mirth L, Popovic Z, Katehi L P B and Whitaker J F 2001 Active-amplifier-array diagnostics using high-resolution electrooptic field mapping IEEE Trans. Microw. Theory Tech. 49 849-57

[4] Reano R M, Yang K, Whitaker J F and Katehi L P B 2001 Simultaneous measurements of electric and thermal fields utilizing an electrooptic semiconductor probe IEEE Trans. Microw. Theory Tech. 49 2523-31

[5] Yariv A and Yeh P 1984 Optical Waves in Crystals (New York: Wiley) chapter 8

[6] Lee D J and Whitaker J F 2008 A simplified Fabry-Perot electro-optic-modulation sensor IEEE Photon. Technol. Lett. 20 866-8

[7] Wakana S, Ohara T, Abe M, Yamazaki E, Kishi M and Tsuchiya M 2000 Fiber-edge electrooptic/magnetooptic probe for spectral-domain analysis of electromagnetic field IEEE Trans. Microw. Theory Tech. 48 2611-6

[8] Yang K, Katehi L P B and Whitaker J F 2001 Electric-field mapping system using an optical-fiber-based electro-optic probe IEEE Microw. Wireless Comp. Lett. 11 164-6

[9] Togo H, Shimizu N and Nagatsuma T 2007 Near-field mapping system using fiber-based electro-optic probe for specific absorption rate measurement IEICE Trans. Electron. E90-C 436-442

[10] Chandani S M 2006 A fiber-based probe for electrooptic sampling IEEE Photon. Technol. Lett. 18 1290-2

[11] Le Quang D, Erasme D and Huyart B 1993 Fabry-Perot enhanced real-time electro-optic probing of MMICs Electron. Lett. 29 498-99

[12] Vickers A J, Tesser R, Dudley R and Hassan M A 1997 Fabry-Perot enhancement electro-optic sampling Opt. Quantum Electron. 29 661-9

[13] Mueller P O, Alleston S B, Vickers A J and Erasme D 1999 An external electrooptic sampling technique based on the Fabry-Perot effect IEEE J. Quantum Electron. 35 7-11

[14] Mitrofanov O 2003 Laser excess noise reduction in optical phase-shift measurements Appl. Opt. 42 2527-31

[15] Mitani S, Yamazaki E, Kishi M and Tsuchiya M 2003 EDFA-enhanced sensitivity of RF magneto-optical probe Int. Topical Meeting Microw. Photon. 10-12 255-8

[16] Sasagawa K and Tsuchiya M 2006 Modulation depth enhancement for highly sensitive electro-optic RF near-field measurement Electron. Lett. 42 1357-8

[17] Lee D J, Kang J J, Chen C C and Whitaker J F 2008 Vector near-field measurement system using an electro-optic microcavity and electrical downconversion IEEE MTT-S Int. Microwave Symp. Digest pp 1589-92

[18] Mitrofanov O, Gasparyan A, Pfeiffer L N and West K W 2005 Electro-optic effect in an unbalanced AlGaAs/GaAs microresonator Appl. Phys. Lett. 86202103

[19] Yang K, Yook J G, Katehi L P B and Whitaker J F 2000 Electrooptic mapping and finite-element modeling of the near-field pattern of a microstrip patch antenna IEEE Trans. Microw. Theory Tech. 48 228-94

[20] Kuo W K, Pai C H and Huang S L 2001 Tech Dig. 4th Pacific Rim Conf. on Lasers and Electro-Optics pp 474-5

[21] Sasagawa K, Tsuchiya M and Izutsu M 2005 Electrooptic probing based on photonic downconversion 8th Int. Symp. Contemporary Photon. Tech. Dig. pp 29-30 